\section{Cholecystocutaneous abscess: A rare presentation of a common problem}

\author{
Yuni Ongso, ${ }^{1}$ Han Nien Beh ${ }^{2}$ \\ ${ }^{1}$ Joondalup Health Campus, WA; \\ ${ }^{2}$ Osborne Park Hospital, WA, Australia
}

\begin{abstract}
Fistulating cholecystocutaneous abscess is a known rare complication of chronic gallstones disease. In these modern days, this complication is exceedingly rare due to early diagnosis with radiological imaging, use of broad spectrum antibiotics and early surgical management of biliary tract disease. Here we present a case of an 89-yearold with cholecystocutaneous abscess that was mistaken for simple subcutaneous abscess. The management include initial control of subcutaneous abscess and use of antibiotics followed by definitive surgical excision of gall bladder and the fistula tract.
\end{abstract}

\section{Introduction}

Fistulating cholecystocutaneous abscess is a rare complication of chronic calculus cholecystitis. In modern clinical medicine, this complication is exceedingly rare due to early radiological diagnosis, availability of broad spectrum antibiotics, and early surgical management of biliary tract disease. Here, we present the case of an 89-year-old man with cholecystocutaneous abscess that was initially mistaken for simple subcutaneous abscess.

\section{Case Report}

The case patient was an 89-year-old man who presented with a persistently draining wound on his right anterolateral chest near the costochondral margin, in the area between the 8 th and 9th ribs. Eight weeks prior, the patient had been seen by his local family doctor for a tender area of swelling on his lower right anterolateral chest. At that time, the patient reported having fallen onto the area 1 week before. Given the history of local trauma, the area of swelling was initially believed to be an infected hematoma, and the patient underwent incision and drainage under local anesthetic. Following this procedure, however, the wound continued to drain purulent material without resolution.
The patient had a prior history of cholelithiasis and recurrent cholecystitis, which had previously been managed conservatively with antibiotics due to his multiple medical comorbidities, including ischemic heart disease with previous acute myocardial infarction and placement of cardiac stents, congestive cardiac failure, type II diabetes, history of transient ischemic attack, chronic obstructive pulmonary disease (COPD), and bilateral carotid artery stenosis. His current medications included aspirin, clopidogrel, carvedilol, atorvastatin, ramipril, glyceryl trinitrate (GTN) patch, and tiotropium.

Physical examination revealed a 5-mm wound on the right lower chest wall and right upper quadrant abdominal tenderness. There was no surrounding skin erythema and no palpable mass. Laboratory testing demonstrated a white blood cell count of $6.5 \times 10^{9} / \mathrm{L}$ and C-reactive protein of 30 $\mathrm{mg} / \mathrm{L}$. Liver function tests were within normal limits. Initial ultrasound (US) of the chest and abdominal wall showed a complex collection likely connecting to a deeper intra-abdominal collection. The patient then underwent computed tomography (CT)
Correspondence: Yuni Ongso, Joondalup Health Campus, Corner of Grand Boulevard and Shenton Avenue, Joondalup, WA 6027, Australia.

Tel.: +61.89400.9400.

E-mail: y.ongso@me.com

Key words: Cholecystocutaneous abscess, cholecystocutaneous fistula.

Contributions: the authors contributed equally.

Conflict of interest: the authors declare no potential conflict of interest.

Received for publication: 21 September 2017. Revision received: 3 November 2017.

Accepted for publication: 4 November 2017

This work is licensed under a Creative Commons Attribution NonCommercial 4.0 License (CC BY-NC 4.0).

(C) Copyright Y. Ongso and H. Nien Beh, 2017 Licensee PAGEPress, Italy

Gastroenterology Insights 2017; 8:6899 doi:10.4081/gi.2017.6899

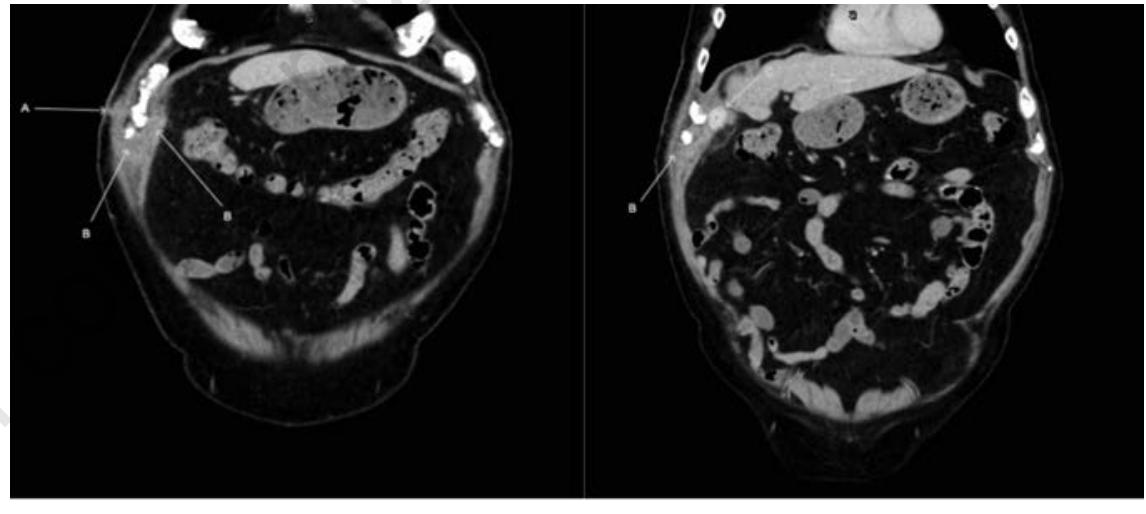

Figure 1. Computed tomography scan of the abdomen. Arrows point to the subcutaneous collection (A) and a gallstone (C) within the suspected fistula tract (B).

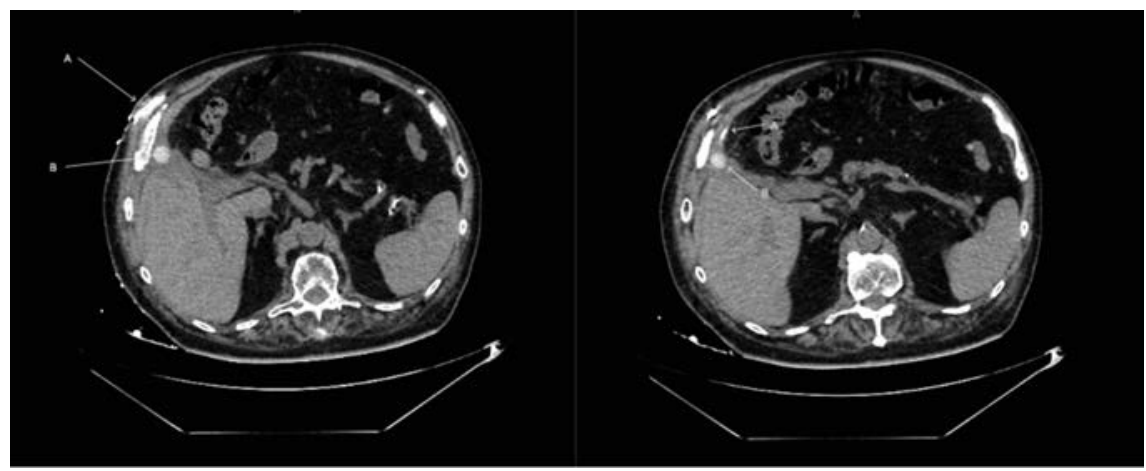

Figure 2. Computed tomography fistulogram. The injected contrast (A) highlights the path of the fistula tract toward the gallstone (B). 
imaging of the abdomen, which demonstrated cholecystitis with a possible fistulous tract between the gallbladder and anterior abdominal wall with a connection to the skin surface, with a gallstone seen within this tract (Figure 1). A CT fistulogram was then performed which confirmed the diagnosis of cholecystocutaneous fistula (Figure 2). Following a thorough medical workup and optimization, the patient underwent laparoscopic cholecystectomy with excision of the fistula tract. The fistula wound was permitted to heal by secondary intention. The patient recovered well following the operation. He was discharged home with daily dressing changes to the fistula wound site. On clinical exam 6 weeks after surgery, the fistula wound had healed completely.

\section{Discussion and Conclusions}

Fistulating cholecystocutaneous abscess is a rare complication of chronic gallstone disease. Even more rarely, it can be due to underlying adenocarcinoma of the gallbladder, a diagnosis which must also be considered in these cases. ${ }^{1}$ Fistulating cholecystocutaneous abscess develops when inflammatory adhesions have formed between the gallbladder and abdominal wall, followed by subsequent perforation of the gallbladder wall at this point of contact and discharge of abscess material into the abdominal wall. Over time, the abscess can track through the skin, forming a fistula tract between the gallbladder and the skin surface. The right upper quadrant of the abdomen is most commonly reported fistula location, while other less frequently reported sites include the epigastrium, right lower quadrant, umbilicus, and gluteal regions. ${ }^{2}$

Fistulating cholecystocutaneous abscess occurs most frequently in geriatric populations, particularly in patients older than 70 years, but this condition has been reported in a patient as young as 30 years old. ${ }^{3}$ When the diagnosis is suspected, imaging modalities to consider include US, CT, and CT fistulogram, which can be used to confirm the diagnosis if there is a cutaneous opening. ${ }^{4}$ Management of cholecystocutaneous abscess includes initial systemic control of sepsis with antibiotics and local control of abscess by incision and drainage, if there has not been spontaneous discharge. In medically unfit patients who cannot undergo general anesthesia, a conservative approach with ongoing management of the gallbladder fistula wound may be appropriate. In a review article published in 1949, 6 out of 37 patients were reported to have spontaneous healing of their fistula wounds. ${ }^{2}$ For patients who are medically fit, definitive surgical excision of the gallbladder and fistula tract should be performed. Of note, in case reports published in the last 50 years, only two cases were successfully managed using laparoscopic techniques. ${ }^{5,6}$ Many patients in these reported cases underwent either planned open procedures or laparoscopic converted to open cholecystectomies.

In summary, cholecystocutaneous abscess should be considered in the differential diagnosis for patients with a right lower chest or upper abdominal mass, especially if the patient has a history of recurrent right upper quadrant pain. Similarly, a chronic draining wound in the right upper quadrant should prompt consideration of the diagnosis of cholecystocutaneous fistula. Definitive surgical excision of the gallbladder and fistula is the treatment of choice. However, conservative management can be considered for medically frail patients.

\section{References}

1. Sodhi K, Athar M, Kumar V, et al. Spontaneous cholecystocutaneous fistula complicating carcinoma of the gall bladder: a case report. Indian J Surg 2012;74:191-3.

2. Henry CL, Ort TG Jr. Spontaneous external biliary fistulas. Surgery 1949;26:641-6.

3. Gossage JA, Forshaw MJ, Stephenson J, Mason R. Spontaneous cholecystocutaneous fistula. J Surgery 2004;2:523.

4. Ulreich S, Henken EM, Levinson ED. Imaging in the diagnosis of cholecystocutaneous fistulae. J Can Assoc Radiol 1983;34:39-41.

5. Malik AH, Nadeem M, Ockrim J. Complete laparoscopic management of cholecystocutaneous fistula. Ulster Med J 2007;76:166-7.

6. Kumar SS. Laparoscopic management of a cholecystocutaneous abscess. Am Surg 1998;64:1192-4. 